Check for updates

Cite this: Chem. Commun., 2021, 57, 1663

Received 21st December 2020 Accepted 13th January 2021

DOI: $10.1039 / \mathrm{d} 0 \mathrm{cc} 08283 \mathrm{j}$

rsc.li/chemcomm

\section{A simple Hückel model-driven strategy to overcome electronic barriers to retro-Brook silylation relevant to aryne and bisaryne precursor synthesis $\dagger$}

\author{
Edward A. Neal, (D)* A. Yannic R. Werling (D) $\ddagger$ and Christopher R. Jones (D)*
}

\begin{abstract}
ortho-Silylaryl triflate precursors (OSATs) have been responsible for many recent advances in aryne chemistry and are most commonly accessed from the corresponding 2-bromophenol. A retro-Brook $O$ - to $C$-silyl transfer is a key step in this synthesis but not all aromatic species are amenable to the transformation, with no functionalized bisbenzyne oSATs reported. Simple Hückel models are presented which show that the calculated aromaticity at the brominated position is an accurate predictor of successful retro-Brook reaction, validated synthetically by a new success and a predicted failure. From this, the synthesis of a novel difunctionalized bisaryne precursor has been tested, requiring different approaches to install the two $C$-silyl groups. The first successful use of a disubstituted $o$-silylaryl sulfonate bisbenzyne precursor in Diels-Alder reactions is then shown.
\end{abstract}

Arynes are a class of highly reactive electron-deficient intermediate derived from an arene or heteroarene with two vacant positions, usually ortho to each other. The reactions of arynes generally involve the introduction or extension of a $\pi$-system and have been extensively reviewed. ${ }^{1}$ These transformations are particularly useful in the pharmaceuticals industry and in $\pi$-extended polyaromatic hydrocarbon (PAH) material production for sensing, energy generation and other applications. ${ }^{1 a, c}$

Classical methods of aryne generation typically required the use of strong bases, sodium metal, pyrophoric tert-butyllithium or potentially explosive ortho-diazocarboxylate precursors, as well as more extreme temperatures. In contrast, ortho-silylaryl triflate precursors (oSATs) operate under milder reaction conditions and possess a more attractive safety profile, ${ }^{2 a}$ which has led to their widespread uptake. ${ }^{1,2 b-d}$ Originally developed by Kobayashi in $1983,{ }^{3 a} o$-(trimethylsilyl)phenyl triflate $\mathbf{5}$ reliably affords benzyne upon treatment with either fluoride or carbonate base. ${ }^{3 b}$ In 2002 Peña et al. improved the synthesis of oSAT 5 through the initial

School of Biological and Chemical Sciences, Queen Mary University of London, Mile End Road, London,UK.E-mail: e.a.neal@qmul.ac.uk, c.jones@qmul.ac.uk $\dagger$ Electronic supplementary information (ESI) available. See DOI: 10.1039/ d0cc08283j

\$ These authors contributed equally to this work. silylation of 2-bromophenol 1 with hexamethyldisilazane (HMDS) (Scheme 1a). ${ }^{4}$ Subsequent halogen-metal exchange then triggered instantaneous retro-Brook $O$ - to $C$-silyl transfer ( 3 to 4 ) prior to triflation.

Whilst this synthetic strategy permits access to many aryne and heteroaryne precursors ${ }^{6}$ with wide functional group tolerance, it is not universal. In some instances, longer routes are needed, such as Kobayashi's original approach ${ }^{3 a}$ via $C, O$-disilylation, $O$-desilylation, then triflation. ${ }^{7}$ This method was used by Duong in 2003 to first produce 1,4-bisbenzyne oSAT precursor $8^{5 b}$ before the successful retro-Brook optimization by Pavliček et al. in 2015 (Scheme 1b). ${ }^{5 c}$ 1,4-Bisbenzynes from 8 have appeared in around 15 papers, ${ }^{5,8}$ as well as a recent trisaryne $o$ SAT from its triphenylene trimer, ${ }^{5 d}$ yet no additionally substituted examples of oSAT bisbenzynes have been developed.

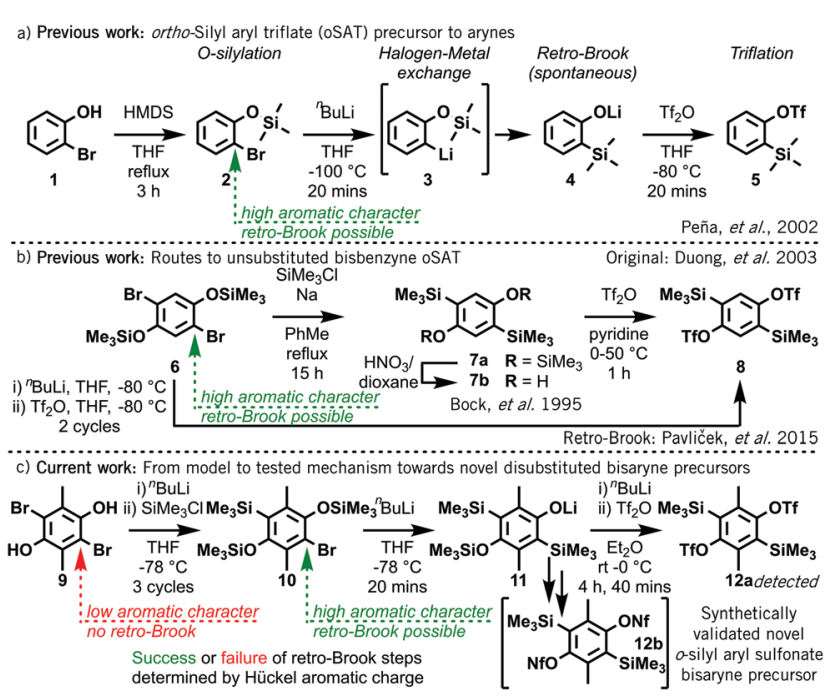

Scheme 1 (a) Synthesis of OSAT benzyne precursor $\mathbf{5}$ from 2-bromophenol $1{ }^{4}$ (b) Approaches to unsubstituted bisbenzyne OSAT precursor $\mathbf{8}^{5 a-c}$ (c) This work: novel route towards challenging disubstituted bisbenzyne precursors based on simple Hückel aromaticity models. 
The different methods of formation of bis/trisarynes have been reviewed by Shi, et al. and usually require more forceful conditions than oSATs, thus limiting functional group tolerance. ${ }^{1 d}$ This presents unrealized potential for the rapid generation of functionalized PAHs from bisbenzyne precursors.

In this work, we demonstrate that an electronic barrier is responsible for the failure of the retro-Brook step during the formation of certain oSATs and that a fast and accessible Hückel calculation provides a reliable indication of success. We then validate this model by experimentally confirming three predicted outcomes: (1) a successful new approach to a known benzyne oSAT precursor; (2) the failure of the key step towards a 9,10-phenanthryne oSAT precursor; ${ }^{9}$ and (3) the development of a synthetic route to a novel difunctionalized bisaryne precursor 12, wherein only one of the two $C$-silyation steps proceeds via a retro-Brook rearrangement (Scheme 1c).

We hypothesized that $o$-bromophenyl silyl ethers, such as 2, should undergo halogen-metal exchange then retro-Brook rearrangement if an aromatic charge is present at the brominated position. To this end, Hückel aromatic charges were calculated on MM2 energy-minimized models of a wide range of bromoaryl silyl ether intermediates ( 2 and $\mathbf{1 3 a}-\mathbf{q})$ towards reported or potential oSAT aryne precursors (Fig. 1). ${ }^{10}$

As a benchmark, the intermediate (2) towards unsubstituted benzyne oSAT 5 has an aromatic charge of $+0.056(\mathrm{ESI}, \dagger \mathrm{S} 2) .^{3 a}$ The sign of the charge was not important, as 1,3-benzodioxole derivative 13a, reported to undergo successful retro-Brook reaction, ${ }^{6 a}$ was calculated at -0.029 . A range of literature examples $\mathbf{1 3} \mathbf{c}-\mathbf{1 3} \mathbf{m}^{7,11 a-i}$ were found to possess charges comparable to benchmark 2 , with just 4,5 -difluoro $13 \mathrm{e}^{11 a}$ showing a significantly lower value $(+0.005)$. In light of this difference, it is noteworthy that several mechanisms for halogen-metal exchange have been proposed. ${ }^{12}$ The model predicted that 3,6-dimethoxy 13n $(-0.011)$ should also undergo retro-Brook rearrangement to the known aryne precursor $13 z$, originally accessed via $C$-deprotonation with ${ }^{n} \mathrm{BuLi}$ and direct $C$-silylation of the 2,5-dimethoxyphenol. ${ }^{11 j}$ Pleasingly, this

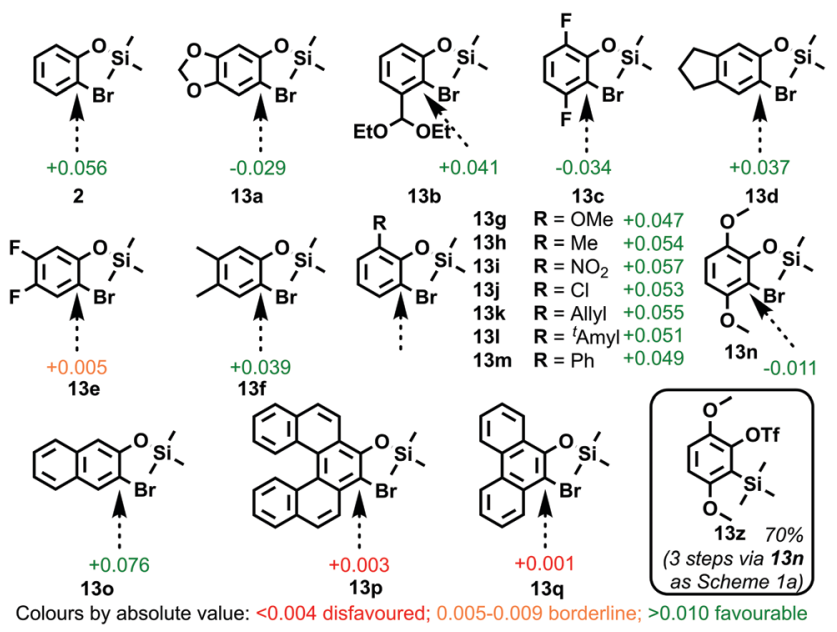

Fig. 1 Calculated Hückel aromatic charges at brominated positions in halogen-metal exchange intermediates for the synthesis of a range of aryne precursors (MM2 in PerkinElmer Chem3D). ${ }^{10}$ novel retro-Brook application proved successful (70\% yield; $\mathrm{ESI}, \dagger \mathrm{S} 3)$.

We next considered the extended aromatic precursors 13o-q, with naphthalene derivative $130^{11 k}$ found to possess a high aromatic charge (+0.076). Interestingly, [5] helicene $13 \mathbf{p}^{11 l}$ also successfully undergoes retro-Brook rearrangement but displays a significantly lower charge $(+0.003)$. As Hückel approximations presume a planar aromatic system, twisted systems such as 13p appear to represent a limitation of our model. By contrast, the planar 9,10-phenanthryne precursor intermediate 13q has a near-zero aromatic charge $(+0.001)$, which suggests the retro-Brook reaction to be disfavoured $;{ }^{9}$ indeed, the synthesis of the corresponding phenanthryne oSAT precursor described by Peña et al. in 2000 did not involve this transformation. ${ }^{13 a}$ According to Clar's Rule, ${ }^{14}$ the two peripheral rings in phenanthrene are more aromatic than the central ring, allowing the 9,10-bond to be functionalized in a manner similar to non-aromatic double bonds. ${ }^{15}$ Even when conjugated alkenes have been exposed to conditions analogous to the retro-Brook reaction, halogen retention was preferred over halogen-metal exchange. ${ }^{16}$

With a view to accessing novel functionalized bisbenzyne oSATs we decided to apply our Hückel model to test the viability of a retro-Brook-based synthetic approach towards paradimethylated bisxylyne oSAT 12. As only small positive inductive effects are present, the steric encumbrance common to all pendant substituents can be tested for, while minimizing electronic effects specific to each. Investigations started by modelling the unfunctionalized bisbenzyne oSAT precursor 7 that was accessed via retro-Brook rearrangement by Pavliček et $a l .{ }^{5 c}$ The MM2 energy-minimized models of post- $O$-silylation intermediates 13r and 13s confirmed that aromatic character was maintained in both brominated positions (Fig. 2). Although calculations on the xylyne precursor $13 \mathbf{t}^{17}$ revealed suitable aromatic character $(+0.026)$, significant deactivation was found for $O$-silylated bisxylyne prototype intermediates $\mathbf{1 3 \mathbf { u }}$ and 13v. Intriguingly, if the first silyl transfer were to be forced, or silylation achieved via other means, then potential intermediates $\mathbf{1 3 w}$ and $\mathbf{1 0}$ for the second stage both have a significant Hückel charge $(+0.032)$. This would suggest that the second silyl transfer should be possible by retro-Brook. Elsewhere, sizeable aromatic charges were calculated for 1,3-diyne
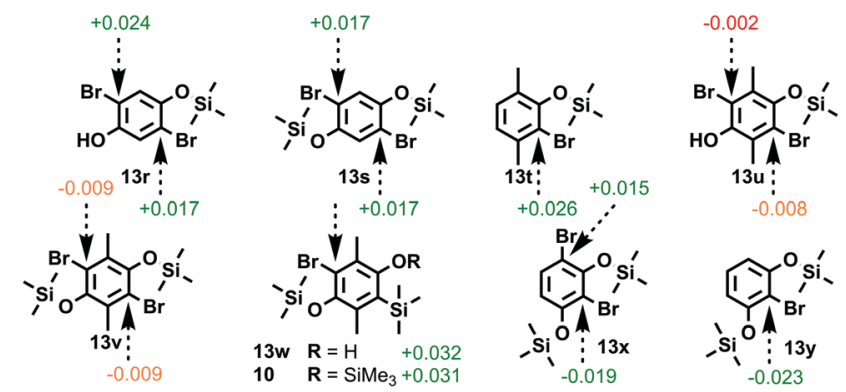

Fig. 2 Hückel aromatic charges on brominated positions in halogenmetal exchange intermediates for a range of literature bisbenzyne precursors and prototype models. (MM2 in PerkinElmer Chem3D) ${ }^{10}$ 
precursors 13x $(-0.019$ and +0.015$)$ and $13 y(-0.023)$, in agreement with the reported retro-Brook syntheses. ${ }^{11 m, n}$

Encouraged by the ability of the Hückel model to predict successful retro-Brook syntheses, we experimentally investigated the two outliers: 9,10-phenanthryne and bisxylyne. The phenanthryne precursor $\mathbf{1 5}$ was first reported by Peña et al., starting from 9-bromo-10-phenanthrol 14 and prepared using a $C, O$-disilylation method. ${ }^{13 a}$ We successfully repeated this on a gram scale, using an in situ solvent-exchange step to remove impurities and increase the yield by $8 \%$ (Scheme $2 \mathrm{a}$ ). ${ }^{13}$

To test our aromaticity model, attempts were made to form oSAT 15 from 14 using HMDS and a retro-Brook reaction, which although reported once ${ }^{4}$ - failed in line with our predictions $\left(\mathrm{ESI}, \dagger\right.$ S5). ${ }^{9}$ To provide sufficient material for these tests, an improved synthesis of $\mathbf{1 4}$ from phenanthrene $\mathbf{1 6}$ was developed (Scheme 2b). Interestingly, the bromination of $\mathbf{1 6}$ at C-9 and C10 would not usually be possible at an aromatic carbon without Lewis acid catalyst. ${ }^{15}$ Our updated synthesis of $\mathbf{1 5}$ has: (a) full contemporary purification and characterization; (b) replaces toxic $\mathrm{CCl}_{4}$ with $\mathrm{CHCl}_{3}$; (c) a 76\% yield of 19 from 18 (cf. $48 \%$ ); and (d) a 34\% yield of 15 at gram scale over five steps from 16 (ESI, $\dagger$ S4). ${ }^{13 a, 18}$ Finally, a quantitative Diels-Alder reaction between 15 and furan was successfully repeated to form adduct $\mathbf{2 0}$ (Scheme 2 inset), validating its suitability as an aryne precursor (ESI, $\dagger$ S5).$^{19}$

Attention now turned to the synthesis of 1,4-bisxylyne $o$ SAT prototype 12a. Our model suggests that the first prospective $O$ - to $C$-silyl transfer in intermediate $\mathbf{1 3 v}$ is disfavoured, whereas a second $C$-silyl group could be installed via a retro-Brook reaction using intermediate $\mathbf{1 3 w}$ or $\mathbf{1 0}$. To probe this prediction, 2,5-dimethylbenzoquinone $\mathbf{2 1}$ was reductively brominated to form 2,5-dibromo-3,6-dimethylhydroquinone 9 (Scheme 3a; $\mathrm{ESI}, \dagger \mathrm{S} 6)$. Attempts to produce bisxylyne oSAT prototype 12a directly from 9 and HMDS via a retro-Brook reaction afforded no product, only crude mixtures highly prone to oxidation (ESI, $\dagger$ S7). Even when forcing conditions were used to access $O$-silyl intermediate $13 \mathbf{v}$, subsequent sodiation ${ }^{5 a}$ to initiate retro-Brook silyl transfer gave an insoluble mixture with no target.

Having verified the fates of intermediates 9 and $13 \mathbf{v}$, a synthetic route towards 12a was developed based on our model. Initial $O, O^{\prime}$-disilylation and $C$-silylation of $\mathbf{9}$, as for phenanthryne oSAT 15, was proposed to access intermediate aryl silane $\mathbf{1 0 .}$

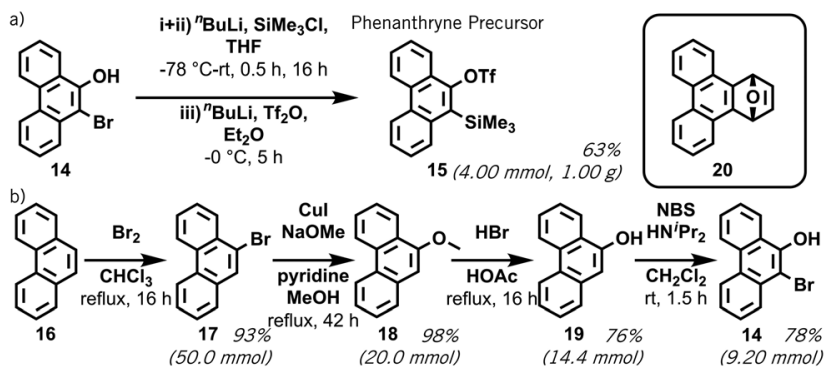

Scheme 2 Synthesis of 9,10-phenanthryne precursor 15: our reported improvements in yield and scale for (a) the final step; (b) the conversion of phenanthrene to precursor starting material 9-bromo-10-phenanthrol 14. This does not involve a retro-Brook step.
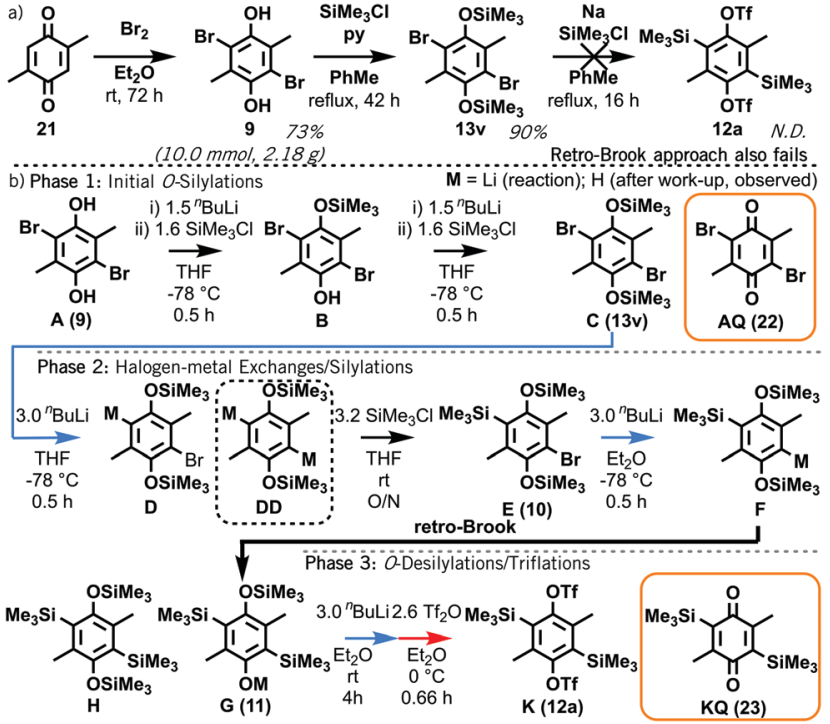

Scheme 3 (a) Reductive bromination of 2,5-dimethyl-1,4-benzoquinone 21 with subsequent failed attempts to form a bisxylyne oSAT prototype 12a; (b) abridged putative mechanism of the formation of 12a (K) from 9 (A). Full scheme in ESI, $\uparrow$ S9.

Subsequent retro-Brook reaction and triflation would lead to oSAT 12a (Scheme 3b; ESI, $\dagger$ S8-9). Reaction aliquots were analysed at each stage by NMR (ESI, $\uparrow$ S14) and GC/MS (ESI, $\dagger$ S18). The $O$-silylations (Phase 1 ) succeeded, although disilylated 13v (C) was observed after the first step and $C$-lithiated $\mathbf{D}$ from halogen-metal exchange after the second, due to excess ${ }^{n} \mathrm{BuLi}$.

Crucially, no retro-Brook reactions were observed with D despite the excess lithiation, as confirmed by a separate test on isolated 13v (ESI, $\dagger \mathrm{S} 10)$. Following the first $C$-silylation step (Phase 2), C, $C^{\prime}$-dilithiated DD was present despite overnight stirring at room temperature with chlorosilane. This suggests a significant barrier to intermolecular $C$-silylation ortho to $O$-silylated species precluding a first retro-Brook (Fig. 3, top). Phenolic signals in the ${ }^{1} \mathrm{H}$ NMR spectrum confirm that the large triply-silylated species must be $C, C^{\prime}, O$-isomer $\mathbf{G}(\mathbf{1 1})$, rather than $\mathbf{F}$. Hence, a retro-Brook reaction on the second side is successful, in line with our mechanistic model. After solvent exchange and further ${ }^{n} \mathrm{BuLi}$, without further silylation, the next aliquot contains only $\mathbf{G}(\mathbf{1 1}), \mathbf{F}$ and tetrasilylated $\mathbf{H}$

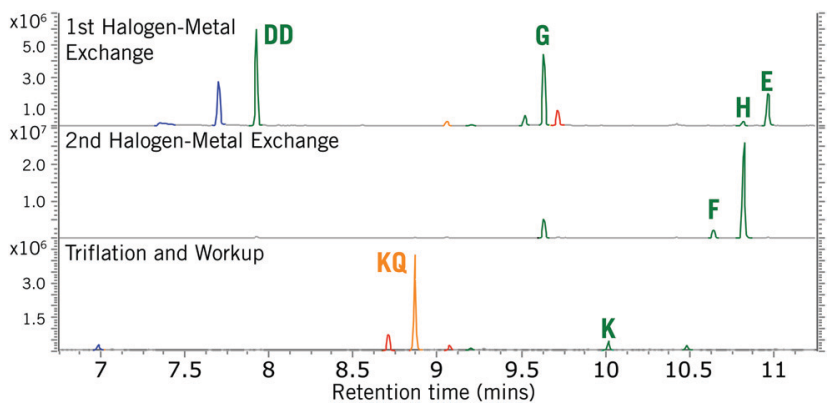

Fig. 3 Partial stack plot of GC/MS aliquots from the formation of bisxylyne OSAT prototype 12a (K). Letters correspond to Scheme 3b. 


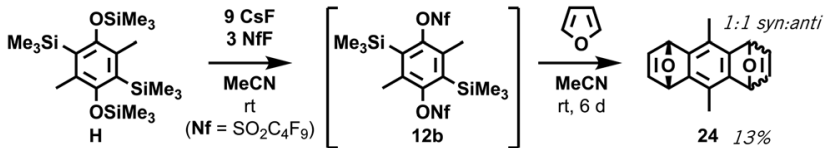

Scheme 4 Successful bisxylene formation from transient ortho-silylaryl nonaflate $\mathbf{1 2 b}$ to form adduct $\mathbf{2 4}$ by in situ Diels-Alder reaction. See ESI, $\dagger$ S12.

(Fig. 3, middle). Here, the 30 minute reaction time allows $\mathbf{E}$ (10) to undergo halogen-metal exchange to afford $\mathbf{F}$ but retro-Brook to $\mathbf{G}$ (11) cannot complete; $\mathbf{H}$ can only form from $O$-silyl exchanges. Unfortunately, after triflation and work-up (Phase 3), quinone KQ (23) predominated (Fig. 3, bottom, 41\% isolated yield); despite this, $C, C^{\prime}$-disilylated $\mathbf{K}(\mathbf{1 2 a})$ is detected in situ and the oxidation product successfully confirms our assignments. $\mathbf{H}$ was later isolated in $74 \%$ yield but attempts to introduce the triflate only produced KQ (23, 56\% yield). This sensitivity of the triflates suggests this may be an inherent limitation of functionalized bis-oSAT precursors. However, transient $o$-silylaryl nonaflates such as $\mathbf{1 2 b}$ (putative structure, accessed from air-stable $\mathbf{H}$ ) are also aryne precursors ${ }^{9}$ and pleasingly the subsequent Diels-Alder reaction of furan with the bisaryne proceeded successfully in $13 \%$ yield (Scheme 4 ).

In summary, we have developed a quick and facile MM2/ Hückel modelling strategy to test the success of retro-Brook $O$ to $C$-silyl transfer relevant to aryne precursor synthesis. A novel application (13z) and an expected failure (15) were validated experimentally. Next, a route towards 1,4-bisxylyne precursors $\mathbf{1 2 a} / \mathbf{b}$ was developed with a retro-Brook step for just one $C$-silylation. This is the first $C, C^{\prime}$-disubstituted $o$-silylaryl sulfonate bisbenzyne precursor: with appropriate substituents, many previously elusive bisaryne precursors may now be accessible.

We are grateful to the EPSRC (EP/M026221/1, CRJ) and QMUL (teaching laboratory technician/teaching fellow posts for EAN; studentship to AYRW) for financial support. We thank the National Mass Spectrometry Facility at Swansea University.

\section{Conflicts of interest}

There are no conflicts to declare.

\section{Notes and references}

1 (a) H. H. Wenk, M. Winkler and W. Sander, Angew. Chem., Int. Ed., 2003, 42, 502-528; (b) D. Pérez, D. Peña and E. Guitián, Chem. - Eur. J., 2013, 5981-6103; (c) S. S. Bhojgude, A. Bhunia and A. T. Biju, Acc. Chem. Res., 2016, 49, 1658-1670; (d) J. Shi, Y. Li and Y. Li, Chem. Soc. Rev., 2017, 46, 1707-1719; (e) T. Roy and A. T. Biju, Chem. Commun., 2018, 54, 2580-2594; $(f)$ D. B. Werz and A. T. Biju, Angew. Chem., Int. Ed., 2019, 59, 3385-3398.

2 Recent cases: (a) A. V. Kelleghan, C. A. Busacca, M. Sarvestani, I. Volchkov, J. M. Medina and N. K. Garg, Org. Lett., 2020, 22, 1665-1669; (b) J. Xu, S. Li, H. Wang, W. Xu and S. Tian, Chem. Commun., 2017, 53, 1708-1711; (c) R. Fan, B. Liu, T. Zheng, K. Xu, C. Tan, T. Zeng, S. Su and J. Tan, Chem. Commun., 2018, 54, 7081-7084; (d) H. Takikawa, A. Nishii, H. Takiguchi, H. Yagishita, M. Tanaka, K. Hirano, M. Uchiyama, K. Ohmori and K. Suzuki, Angew. Chem., Int. Ed., 2020, 59, 12440-12444.

3 (a) Y. Himeshima, T. Sonoda and H. Kobayashi, Chem. Lett., 1983, 1211-1214; (b) S. Yoshida, Y. Hazama, Y. Sumida, T. Yano and T. Hosoya, Molecules, 2015, 20, 10131-10140.
4 D. Peña, A. Cobas, D. Pérez and E. Guitián, Synthesis, 2002, $1454-1458$

5 (a) H. Bock, S. Nick, C. Näther and K. Ruppert, Z. Naturforsch., 1995, 50, 595-604; (b) H. M. Duong, M. Bendikov, D. Steiger, Q. Zhang, G. Sonmez, J. Yamada and F. Wudl, Org. Lett., 2003, 5, 4433-4436; (c) N. Pavliček, B. Schuler, S. Collazos, N. Moll, D. Pérez, E. Guitián, G. Meyer, D. Peña and L. Gross, Nat. Chem., 2015, 7, 623-628; (d) I. Pozo, D. Peña, E. Guitían and D. Pérez, Chem. Commun., 2020, 56, 12853-12856.

6 (a) Y. Sato, T. Tamura and M. Mori, Angew. Chem., Int. Ed., 2004, 43, 2436-2440; (b) F. I. M. Idiris, C. E. Majesté, G. B. Craven and C. R. Jones, Chem. Sci., 2018, 9, 2873-2878.

7 A. B. Smith and W.-S. Kim, Proc. Natl. Acad. Sci. U. S. A., 2011, 108, 6787-6792.

8 Selected examples: (a) R. Shintani, H. Otomo, K. Ota and T. Hayashi, J. Am. Chem. Soc., 2012, 134, 7305-7308; (b) K. G. U. R. Kumarasinghe, F. R. Fronczek, H. U. Valle and A. Sygula, Org. Lett., 2016, 18, 3054-3057; (c) P. Asgari, U. S. Dakarapu, H. H. Nguyen and J. Jeon, Tetrahedron, 2017, 73, 4052-4061; (d) X. Yang and G. C. Tsui, Chem. Sci., 2018, 9, 8871-8875; (e) W. Xu, X.-D. Yang, X.-B. Fan, X. Wang, C.-H. Tung, L.-Z. Wu and H. Cong, Angew. Chem., Int. Ed., 2019, 58, 3943-3947; $(f)$ Most recent of seven works: I. Pozo, Z. Majzik, N. Pavliček, M. Melle-Franco, E. Guitián, D. Peña, L. Gross and D. Pérez, J. Am. Chem. Soc., 2019, 141, 15488-15493.

9 T. Ikawa, S. Masuda, H. Nakajima and S. Akai, J. Org. Chem., 2017, 82, 4242-4253. See ESI $\dagger$ S5.

10 ChemOffice Professional, PerkinElmer Informatics, 2019.

11 (a) V. Singh, R. S. Verma, A. K. Khatana and B. Tiwari, J. Org. Chem., 2019, 84, 14161-14167; (b) Y. Tsuchido, T. Ide, Y. Suzaki and K. Osakada, Bull. Chem. Soc. Jpn., 2015, 88, 821-823; (c) H. Jiang, Y. Zhang, W. Xiong, J. Cen, L. Wang, R. Cheng, C. Qi and W. Wu, Org. Lett., 2019, 21, 345-349; (d) C.-H. Sun, Y. Lu, Q. Zhang, R. Lu, L.Q. Bao, M.-H. Shen and H.-D. Xu, Org. Biomol. Chem., 2017, 15, 4058-4063; (e) K. Nogi, T. Fujihara, J. Terao and Y. Tsuji, J. Org. Chem., 2015, 80, 11618-11623; $(f)$ C. Hall, J. L. Henderson, G. Ernouf and M. F. Greaney, Chem. Commun., 2013, 49, 7602; (g) Y. Zeng, L. Zhang, Y. Zhao, C. Ni, J. Zhao and J. Hu, J. Am. Chem. Soc., 2013, 135, 2955-2958; (h) J. Pan, M. Su and S. L. Buchwald, Angew. Chem., Int. Ed., 2011, 50, 8647-8651; (i) B. Lakshmi, U. Wefelscheid and U. Kazmaier, Synlett, 2011, 345-348; $(j)$ J. George, J. S. Ward and M. S. Sherburn, Org. Lett., 2019, 21, 7529-7533; (k) T.-Y. Zhang, C. Liu, C. Chen, J.-X. Liu, H.-Y. Xiang, W. Jiang, T.-M. Ding and S.-Y. Zhang, Org. Lett., 2018, 20, 220-223; (l) T. Hosokawa, Y. Takahashi, T. Matsushima, S. Watanabe, S. Kikkawa, I. Azumaya, A. Tsurusaki and K. Kamikawa, J. Am. Chem. Soc., 2017, 139, 18512-18521; $(m)$ T. Ikawa, S. Masuda, A. Takagi and S. Akai, Chem. Sci., 2016, 7, 5206-5211; (n) J. Shi, D. Qiu, J. Wang, H. Xu and Y. Li, J. Am. Chem. Soc., 2015, 137, 5670-5673.

12 T. D. Sheppard, Org. Biomol. Chem., 2009, 7, 1043-1052.

13 (a) D. Peña, D. Pérez, E. Guitián and L. Castedo, J. Org. Chem., 2000, 65, 6944-6950; (b) M. Idris, C. Coburn, T. Fleetham, J. MilamGuerrero, P. I. Djurovich, S. R. Forrest and M. E. Thompson, Mater. Horiz., 2019, 6, 1179-1186.

14 E. Clar, Polycyclic Hydrocarbons, Springer, Berlin, 1964.

15 C. A. Dornfeld, J. E. Callen and G. H. Coleman, Org. Synth., 1948, 28, 19-21. Bromine adds across the 9,10-bond prior to rearomatization on heating.

16 (a) T. J. Barton and B. L. Groh, J. Am. Chem. Soc., 1985, 107, 7221-7222; (b) N. Shimizu, F. Shibata and Y. Tsuno, Bull. Chem. Soc. Jpn., 1987, 60, 777-778; (c) L. Duhamel, J. Gralak and B. Ngono, J. Organomet. Chem., 1989, 363, C4-C6; (d) L. Duhamel, J. Gralak and A. Bouyanzer, Tetrahedron Lett., 1993, 34, 7745-7748; (e) L. Duhamel, J. Gralak and B. Ngono, J. Organomet. Chem., 1994, 464, C11-C13.

17 Y. Wang, A. D. Stretton, M. C. McConnell, P. A. Wood, S. Parsons, J. B. Henry, A. R. Mount and T. H. Galow, J. Am. Chem. Soc., 2007, 129, 13193-13200.

18 (a) R. G. R. Bacon and S. C. Rennison, J. Chem. Soc. C, 1969, 312-315; (b) S. Kobayashi, K. Kitamura, A. Miura, M. Fukuda, M. Kihara and M. Azekawa, Chem. Pharm. Bull., 1972, 20, 694-699.

19 M. McKee, J. Haner, E. Carlson and W. Tam, Synthesis, 2014, 1518-1524. 